\title{
Probiotics Supplementation Alleviate Corticosterone- induced Fatty Liver Disease Related With the Changes of Hepatic Lipogenesis and Gut Microbiota Profile in Broiler
}

\author{
Wenqing Mei \\ Nanjing Agricultural University \\ Yuyan Feng \\ Nanjing Agricultural University \\ Zhihao Yao \\ Nanjing Agricultural University \\ Haonan Luo \\ Ridley College \\ Yingdong Ni ( $\nabla$ niyingdong@njau.edu.cn ) \\ Nanjing Agricultural University \\ Ruqian Zhao \\ Nanjing Agricultural University
}

\section{Research}

Keywords: Fatty liver disease, Corticosterone, Probiotic, Gut microbiota, Broiler

Posted Date: June 15th, 2020

DOI: https://doi.org/10.21203/rs.3.rs-35344/v1

License: () (1) This work is licensed under a Creative Commons Attribution 4.0 International License. Read Full License 


\section{Abstract}

Background:Emerging evidencesindicate a close relationship between gut microbiotaand fatty liver disease.It has been suggested that gut microbiota modulation with probiotics improved fatty liver disease in rodents and human, yet it still remains unclear in poultry.

Results: Ninety six one-day-old green-legged chicken were divided into control group (CON) and probiotic group (PB), respectively. Probiotics were administrated throughdrinking water for two weeks from 1-day-old. At $28 \mathrm{~d}$ of age, 16 broilers selectedfromCON or PB group randomly, and receivedvehicle( $15 \%$ ethanol) or CORT(4.0 $\mathrm{mg} / \mathrm{kg}$ )treatmentdaily viasubcutaneous injection for a week to induce fatty liver. At the end of the experiment, broilers from 4 groups,control group(CON), corticosterone group (CORT), probiotic group (PB), PB plusCORT group(CORT\&PB),were slaughtered for sampling and analysis. The results showed that probiotics administration significantly prevented CORT-induced body weight loss $(P<0.05)$, but did not alleviate the reduction of immune organs weight caused by CORT. Compared to CON,broilers in CORT group exhibited a significant increase of triglyceride (TG) levelin both plasma and liver $(P<0.01)$, as well as severe hepatocytic steatosis and hepatocellular ballooning, and accompanied with the up-regulation of hepatic lipogenesis genes expression. However, probiotics supplementation markedly decreased the intrahepatic lipid accumulation and steatosis histological score, which was associated withthedown-regulation of sterol regulatory element-binding protein-1 (SREBP1) and acetyl-CoA carboxylase (ACC) mRNA $(P<0.05)$ as well as it protein $(P=0.06)$ expression.Cecal microbiota composition was determined by $16 \mathrm{~S}$ rRNA high-throughput sequencing. Results showed that CORT treatment induced distinct gut microbiota alterations with a decrease of microbial diversity, and anincrease of proteobacteriaabundance $(P<0.05)$. On the contrary, probiotic supplementation increased the beta diversity and increased community richness and diversity index $(P>0.05)$, as well as the abundance of Intestinimonas $(P<0.05)$.

Conclusion: Our results indicate that CORT treatment induced serious fatty liver disease and altered the gut microbiota composition in broilers, however,probiotics supplementation from post-hatching had a beneficial effect on alleviatingfatty liver disease through regulating lipogenic genes expression and increasing gut microbiota diversity andbeneficial bacteria abundanceimbalance.

\section{Background}

Nonalcoholic fatty liver disease (NAFLD) is a burgeoning health problem that affects a considerable proportion of adults in western countries as well as the developing countries [1]. It comprises a spectrum of liver pathology including hepatocellular steatosis, steatohepatitis, fibrosis, and cirrhosis[2]. In chicken, the relative basic lymphatic system and transport of fat in chylomicrons into the portal vein increase their susceptibility to hepatic fat deposition. The accumulation of TG in liver was the main feature of fatty liver disease[3]. Hepatic de novo lipogenesis (DNL) is thought to play a pivotal role in the development of fatty liver disease[4]. The key enzymes in the process of DNL include sterol regulatory element-binding protein-1 (SREBP1), fatty acid synthase (FASN) and acetyl-CoA carboxylase (ACC). Thus, lipogenesis has become a target for the prevention and therapeutic treatment of fatty liver disease.

Glucocorticoid hormones (GCs) are important regulators of lipid metabolism, promoting lipogenesis when treated with chronic exposure[5].For instance, chronic excessive GCs exposure induced whole-body insulin 
resistance, strongly related to metabolic dysfunction including intrahepatic lipid accumulation, intramuscular fat deposition and abdominal adiposity in humans[6, 7].Corticosterone (CORT) is the main active form of GC in chickens. Previous studies have shown that fatty liver disease can be induced by excessive CORT exposure in broilers[8-10].

The relationship between intestinal microbiota dysbiosis and liver diseases has been reported[11].Intestinal microbiota has been proposed as a regulator of energy homeostasis and ectopic fat deposition, which indicated its implications in metabolic diseases[12]. Thus, the change of gut microbiota seems to play an important role in the induction and promotion of liver injury. Although the mechanism of probiotics have not been completed understood, it has been considered to be the possible adjunctive therapy in fatty liver disease due to the numerous beneficial effects such as improving epithelial barrier function, preventing bacterial translocation, inhibiting bacterial mucosal adhesion, and reducing inflammation[12]. Probiotics supplementation can prevent the occurrence and development of fatty liver disease, improve liver steatosis and fibrosis in mice fed with highfat diet (HFD) $[13,14]$. The protective effects of probiotics on fatty liver disease by many pathways including reducing hepatic lipid deposition, endotoxemia and oxidative stress[15]. It's reported that Parabacteroides distasonis can alleviate the disorder of lipid metabolism dysfunction in ob/ob mice and HFD mice, indicating that weight loss, a decrease in hyperglycemia and hyperlipidemia, and the improvement of hepatic steatosis[16].Ritze reported that Lactobacillus rhamnosus $G G$ attenuated liver inflammation and steatosis in high glucose diet-induced NAFLD model mice[17]. A study showed that a dietary supplementation with a probiotic, the Primalac 454, was performed in 3 week old Ross-308 broiler chickens for 4 weeks, which can limit the intensity of the liver fatty disease induced with dietary protein restriction ( $14 \%$ vs $20 \%$ crude protein) [18]. However, the effect of the supplementation of probiotics mixture during the early growth stage of broilers on the gut microbiota profiles and the protective function on fatty liver disease in broilers still awaits for further investigation.

Therefore, the aim of this study was to investigate whether probiotics mixture supplemented from after hatching can prevent CORT-induced fatty liver disease in broilers, and to determine how such effects, if any, are associated with hepatic de novo lipogenesis and gut microbiota composition.

\section{Methods}

\section{Animals and Treatment}

A total of 96 one-day-old Green-legged chicken were purchased from Zhenjiang Wenshi company. All chicks were exposed to continuous illumination and diets and free access to water were supplied ad libitum. The ambient temperature was maintained at $35 \sim 37^{\circ} \mathrm{C}$ during the first 3 days, then gradually decreased by $0.5^{\circ} \mathrm{C}$ every day until reaching a final temperature of $21^{\circ} \mathrm{C}$. Dietary composition is shown in Table 1 . Broilers were randomly divided into control (CON) group and probiotic (PB) group. Each group contained 48 broilers in 6 replicates or cages (8 broilers/cage). PB group chickens were received probiotics $100 \mathrm{mg}$ per chicken respectively via drinking water for two weeks. Probiotics purchased from Jiangsu H.F.Q. TECHNOLOGY CO., LTD., composed of bifidobacterium, lactobacillus acidophilus, streptococcus faecalis and yeast.

Bifidobacterium and lactobacillus acidophilus are not less than $1.0 \times 10^{7} \mathrm{CFU}$, and Streptococcus faecalis and yeast are not less than $1.0 \times 10^{6} \mathrm{CFU}$ per gram. Broilers were fed with the basal diet during the whole 
experiment. After 4 weeks, broilers were randomly divided into four groups $(n=8 /$ group $)$, control group (CON), corticosterone group (CORT), probiotic group (PB),PB and CORT treatment group (CORT\&PB), respectively. Broilers in CON group and PB group received vehicle daily subcutaneous injection of solvent (15\% ethanol). Broilers in CORT group and CORT\&PB group received CORT ( $4.0 \mathrm{mg} / \mathrm{kg}$ body weight) daily subcutaneous injection for 7 days. Corticosterone (C2505) were purchased from Sigma-Aldrich Chemical Co. (St. Louis, MO, USA).

Body weight was recorded from 1 to $35 \mathrm{~d}$ of age. At $36 \mathrm{~d}$ of age, 32 broilers ( 8 broilers each group) were slaughtered. Liver, spleen, fabricius and pectoralis immune organs were weighted. Blood samples were collected, centrifuged at $3500 \mathrm{rmp}$ for $10 \mathrm{~min}$, and plasma samples were separated out and stored at $-20^{\circ} \mathrm{C}$. Subsequently, liver samples were collected into liquid nitrogen and keep at $-80^{\circ} \mathrm{C}$ for further analysis. Liver samples used to make tissue slices were placed in $4 \%$ paraformaldehyde. Cecum contents were collected and stored at $-20^{\circ} \mathrm{C}$.

\section{Histopathology}

Liver specimens was fixed with $4 \%$ formaldehyde-buffered solution and paraffin-embedded, and then sectioned for hematoxylin eosin (HE) staining. Frozen sections of liver were stained with Oil Red. Samples were rated based on the severity of steatosis, and graded $0-4$, indicating none, slight, mild, moderate, and severe, respectively. Steatosis scores were analyzed independently by three veterinarians with reference to a previous publication[8]. In each case, five tissue sections were examined and a numerical score was assigned.

\section{Plasma Biochemical Indicators Measurement and TG concentration in liver}

The concentration of triglyceride (TG) in plasma were measured by an automatic biochemical analyzer (7020, HITACHI, Tokyo, Japan) using commercial kits (E1003; Applygen Technologies Inc., Beijing, China).Hepatic TG were measured using a triglyceride assay kit(E1013; Applygen Technologies Inc., Beijing, China) following the manufacturer's instructions.

\section{Reverse Transcription and Real-time PCR}

Total RNA was isolated from liver samples with the TRIzol reagent (15596026; Invitrogen, Shanghai, China). The concentration and quality of the RNA were assessed by a Nano Drop ND-1000 Spectrophotometer (Thermo Fisher Scientific, Madison, WI, USA). Next, $2 \mu \mathrm{g}$ of total RNA was treated with RNase-Free DNase (M6101; Promega, Madison, WI, USA) and was reverse transcribed according to manufacturer's instructions. Two microliters of diluted cDNA (1:20, v/v) were used for real-time PCR with a Mx3000 P Real-Time PCR System (Stratagene, USA). $\beta$-actin, which is not affected by CORT and PB, was chosen as the reference gene. All primers were synthesized by Tsingke Company (Shanghai, China), and are listed in Table 2 . The $2^{-\triangle \Delta C T}$ method was used to analyze the real-time PCR data[19].

\section{Western blotting analysis}

Total protein was extracted from a 30-mg sample of frozen liver as previously described[20]. A BCA Protein Assay kit(Pierce, Rockford, USA) was used to measure the protein concentration. $40 \mu \mathrm{g}$ sample of protein was separated by 10\% SDS-PAGE gel. Western blot analysis for FASN (AB43451,Abcam, 1:500), ACC (AF6421, 
Affinity, 1:500), SREBP1 (14088-1-AP, Proteintech, 1:500), CPT1A (ab83862, Abcam, 1:200), CPT1B (BS70732, Bioworld, 1:500) were carried out following the manufacturer's instructions. Images were captured by VersaDoc 4000MP system (Bio-Rad, USA) and the band density was analyzed with image j.

\section{Extraction of genome DNA and 16S rRNA sequencing}

Total genome DNA from samples was extracted using CTAB/SDS method. DNA concentration and purity was monitored on $1 \%$ agarose gels. According to the concentration, DNA was diluted to $1 \mathrm{ng} / \mu \mathrm{L}$ using sterile water. 16S rRNA genes of distinct regions (16SV3-V4) were amplified used specific primer with the barcode. Phusion ${ }^{\circledR}$ high fidelity PCR master mix with GC buffer from New England Biolabs and high fidelity enzyme are used for PCR to ensure the efficiency and accuracy of amplification. The PCR product was verified by electrophoresis in $2 \%$ agarose gel, and then recycled by GeneJET ${ }^{\mathrm{TM}}$ Gel Extraction Kit (Thermo Scientific). Sequencing libraries were generated using lon Plus Fragment Library Kit 48 rxns (Thermo Scientific) following manufacturer's instructions and sequenced on an Ion $S 5^{\mathrm{TM}} \mathrm{XL}$ platform. Sequences analysis were performed by Uparse software. Species annotation analysis was measured using mothur method and Silva Database. Data were normalized using a standard of sequence number according to the least sequences. Alpha and Beta diversity was calculated and displayed with QIIME(Version1.7.0) and R software (Version 2.15.3).

\section{Statistical Analysis}

Results are expressed as means \pm SEM and were analyzed by two-way ANOVA using the General Linear Model(GLM) producer in SPSS 20.0 for Windows (IBM Cooperation, Armonk, NY, USA). Data were analyzed using a $2 \times 2$ factorial arrangement with the main effects of CORT and PB treatment. LSD post hoc analysis was used for evaluating differences among specific groups. The level of significant was based on the probability value $P<0.05$

\section{Results}

\section{Body weight and the relative weight of immune organs}

At 28 days of age, there was no significant difference of body weight between CON and PB group $(P>0.05$; Fig. 1). Compared to CON, CORT administration markedly decreased body weight $(P<0.01$; Fig. 1), however, chickens in CORT\&PB group showed higher body weight than those in CORT group $(P<0.05$; Fig. 1).

CORT treatment significantly increased the liver weight and index $(P<0.01$; Table 3$)$, while PB supplementation significantly decreased the liver index $(P<0.05$; Table 3$)$. The weight of immune organs including thymus, spleen and fabricius and their relative weight were significantly decreased by CORT injection compared to CON $(P<0.01$; Table 3). PB supplementation did not alter the immune organs growth $(P>0.05 ;$ Table 3$)$.

\section{Hepatic Histological Analysis}

Hepatic histological sections stained with hematoxylin-eosin (HE) and Oil Red O showed that severe hepatic lipid accumulation was obviously observed in the CORT group; however, only slight vacuolar steatosis was showed in the CORT\&PB group (Fig. 2a and b). As shown in Fig. 2c and d, CORT injection significantly increased the total steatosis score estimated by the HE staining and Oil Red $O$ staining $(P<0.05)$, yet PB treatment had a tendency to decrease it $(P=0.09)$. 


\section{TG concentrations in plasma and the liver}

As shown in Fig. 3, CORT treatment significantly increased the TG concentration in plasma and the liver compared to the control group $(P<0.01)$, and PB administration significantly decreased plasma TG concentration $(P<0.05)$ and tended to reduce the hepatic TG level $(P=0.06)$. The CORT\&PB group showed lower levels of TG in plasma and the liver when compared to the CORT group $(P<0.05)$.

\section{Expression of Genes Related to TG metabolism in the liver}

As shown in Fig. 4, CORT significantly up-regulated hepatic ACC $(P<0.01)$, SREBP1 $(P<0.01)$, FASN $(P<0.05)$, SCD $(P<0.01)$ and DGAT2 $(P<0.05)$ mRNA expression, however, PB treatment markedly down-regulated ACC $(P<0.01)$, SREBP1 $(P<0.05)$, DGAT2 $(P<0.05)$ and FASN $(P=0.08)$ mRNA expression in the liver. Moreover, there was a significant interaction of CORT and PB on hepatic ACC and SREBP1 genes expression $(P<0.05)$ (Fig. 4a and b).

The expression of genes related to lipolysis and TG transport in the liver was also changed by CORT but not PB treatment. CORT significantly down-regulated LPL mRNA expression $(P<0.01)$ but not PPARa expression $(P>$ $0.05)$. There was no significant effect of $P B$ on these two genes expression $(P>0.05 ; \mathrm{Fig} .4 \mathrm{~g}$ and $\mathrm{h})$. CORT significantly up-regulated hepatic APOA1 mRNA expression ( $P<0.01$; Fig. 4j), but did not change CD36 or APOB mRNA expression ( $P>0.05$; Fig. $4 \mathrm{i}$ and $\mathrm{k}$ ). Probiotic significantly down-regulated hepatic APOB mRNA expression $(P<0.05$; Fig. 4k).

\section{Expression of Protein Related to Lipid Metabolism in the Liver}

CORT treatment significantly up-regulated the protein expression of FASN and $\operatorname{ACC}(P<0.01$; Fig. $5 b$ and d), while PB showed a tendency to down-regulate these two proteins expression in the liver $(0.05<P<0.1 ;$ Fig. $5 \mathrm{~b}$ and d). There was a significant interaction between PB and CORT on hepatic ACC protein expression $(P<0.05$; Fig. 5b). CORT injection significantly down-regulated the CPT1A protein expression ( $P<0.05$; Fig. 5f). SREBP1 protein expression was not altered by CORT or PB treatment ( $P>0.05$;Fig. $5 \mathrm{C})$.

\section{The analysis of cecum microbiota composition}

A total of 1,227,484 clean reads were obtained for the bacterial 16S rRNA genes by high-throughput sequencing analysis and 1082 OUTs were obtained by clustering at $97 \%$ identity. The number of common and unique OTUs among four groups was shown in Venn diagram (Fig. 6a), describing sample similarity and overlap intuitively. The PB group had the highest number of unique sequences (49 OTUs), followed by the CORT group (41 OTUs), the CON group (25 OTUs) and CORT\&PB group(23 OTUs)(Fig. 6a). In addition, there were 648 OTUs (approximately $60 \%$ of total OTUs) shared among four groups(Fig. 6a). Rarefaction curve was performed by the number of OTUs and sequences randomly extracted from samples, directly reflecting the rationality of sequencing data. The Fig. $6 \mathrm{~B}$ shows that as the depth of sequencing increases, the curve tends to be flat, indicating that the sequencing results were reasonable, and each sample can truly reflect the microbial composition.

At the phylum level, firmicutes, proteobacteria and Bacteroidetes were the most prominent in four groups(Fig. 6c). We found an obvious phylum-wide shift in the proteobacteria induced by CORT 
challenge(Fig. 6c). The proteobacteria abundance was significantly increased in the CORT group compared with the CON group (Fig. 6c).

The community richness and diversity of cecal microbiota was presented by Chao index, ACE index and Shannon index, Simpson index. CORT treatment reduces the four index, yet this effect was reversed by PB supplementation ( $P>0.05$; Fig. 7a-d). Compared with the CON group, broilers in PB group had higher beta diversity based on weighted unifrac $(P<0.05$; Fig. 7e). The community composition of gut microbiota was analyzed by principal component analysis (PCA). We found that PCA can notably show the significant differences of cecal microbiota composition between four groups(Fig. 7f). The PCA score plot showed that the gut microbiota of the CORT group exhibited a shift along the positive direction of the second principal component (PC2) compared with that of the CON group, and the gut microbiota of the PB group showed a notable shift along the positive direction of the PC2 as well as the positive direction of the first principal component (PC1)(Fig. 7f). Interestingly, bacterial composition in CORT\&PB group was similar to that in the CON group. Collectively, the community composition of gut microbiota altered by the CORT challenge was partly reversed by probiotics administration tracing to be similar to the profile of the control chickens.

Furthermore, the differences species between CORT and CORT\&PB group in genus level were also analyzed by T-test. Compared to the CORT group, the abundance of intestinimonas and caproiciproducens was significantly increased in CORT\&PB group (Fig. $7 \mathrm{~g}$ ).

\section{Discussion}

Fatty liver disease becomes a concern healthy problem in domestic chickens, causing a significant economic lost in poultry industry. Liver is a vital organ for lipid metabolism, particularly in avian species. Lipid drops are usually accumulated in liver tissue, causing hepatic structural disruption and dysfunction, which ultimately leads to liver hypertrophy and fatty liver disease[21]. Therefore, controlling lipid accumulation in the liver can prevent the progression of lipid metabolic disorders[22]. In this study, fatty liver disease was mimicked in broilers by consecutive injection of CORT, showing with the typical characteristics of fatty liver disease as the previous report[10]. However, our results showed that probiotics supplementation from post-hatching for 2 weeks could effectively protect the lost of body weight, and prevent the progress of fatty liver disease induced by CORT in broilers. It's reported that chronic CORT treatment reduces protein synthesis but promotes protein degradation, and finally leading to the loss of body weight in chickens[10,23]. It has been described that probiotic exhibits beneficial effect on body weight gain[24, 25]. Wang et al. [26] found that Bacillus amyloliquefaciens SC06 administration improve HFD-induced poor growth performance. Similarly, our results showed that probiotics supplementation during early growth stage could effectively prevent the loss of body weight.

The significant increase of TG concentration in plasma and liver, as well as histological features including steatosis and vacuolar degeneration by CORT injection in the present study was in accordance with our previous work in chickens subjected to exogenous CORT exposure[9] and in rats exposed to dexamethasone(DEX)challenge [27]. Interestingly, our results clearly showed that these phenotypic characterizations of fatty liver disease in broilers was greatly ameliorated by administration of probiotics mixture administration. Consistent with the results of this study, Wang et al.[16] reported that Parabacteroides distasonis alleviates an increase in intrahepatic triglyceride in high-fat diet (HFD)-fed mice. Yalçin et al. [18]also 
obtained the similar results, indicating that the supplementation of probiotics to broilers play a beneficial effect on alleviating the fatty liver disease. In chickens, fatty acid synthesis primarily occurs in liver, whereas in mammals adipose tissue is the main lipogenic tissue [28]. Four major pathways control the hepatic TG accumulation: TG import via uptaking the dietary TG, de novo lipogenesis, the utilization of TG through fatty acid $\beta$-oxidation (lipolysis), and TG export from the liver [29]. Many studies have confirmed that de novo lipogenesis play a vital role in the progression of fatty liver disease[30]. Previous study indicated that hepatic fat deposition in NAFLD patients mainly attributed to the highly regulated metabolic pathway for the synthesis of fatty acids from ACC[30]. Consistently, the fatty liver disease caused by exogenous glucocorticoids exposure was mainly resulted from the activation of lipogenesis in chickens [9] as well as in rats [27]. In this study, our results indicated that the mRNA expression levels of ACC, the key rate-limiting enzyme of de novo lipogenesis, and SREBP-1, a key transcription factor of downstream of ACC, in the chicken liver were increased by CORT challenge. Notably, probiotic supplementation could alleviate the CORT challenge-mediated activation of the gene expression of lipogenesis in birds. Similar results have been reported by Wang et al.[31] who noted that Lactobacillus johnsonii BS15 treatment could improve lipid metabolism in liver by inhibiting the hepatic fatty acid de novo synthesis, accompanied with down-regulated SREBP-1 and its target genes, FASN and SCD. In addition, although CORT treatment had significant effects on lipolysis and lipid transport, probiotics supplementation exert minimal effects. These results present convincing evidence to support our view that the possible mechanism by which probiotics protect fatty liver disease induced by CORT injection may be associated with diminished gene expression levels of DNL pathway.

Modulation of gut microbiota with probiotics have recently been implemented in the prevention and treatment of several metabolic diseases, such as fatty liver disease[32]. Hamid et al. also confirmed that general microbiota imbalance was linked with NAFLD[33]. Probiotics are considered to normalize gut microbiota and reverse microbial dysbiosis, which could potentially benefit host. Wang et al.[31] reported that dietary supplementation of Lactobacillus johnsonii BS15 improve hepatic lipid metabolism in broilers, accompanied with the lower Firmicutes/Bacteroidetes ratio. A low Firmicutes/Bacteroidetes ratio can be effective against obesity[34]. Here, we indicate that the composition of the gut microbiota seems to play a role for the prevention of fatty liver disease. We showed that the richness and diversity of community decreased in broilers with CORTinduced fatty liver disease. Similar results have been observed in the studies of the subjects suffering from illness, such as obesity[35] and liver cirrhosis[36]. Interestingly, an increase in alpha and beta diversity of gut microbiota was observed after probiotic supplementation, and it was similar to the previous results of Zhou et al.[37], who reported that probiotic treatment could increase Shannon index in HFD rats. Furthermore, we also observed a significant alteration of gut microbiota under the condition of CORT injection. The result of alterations in gut microbiota composition induced by CORT injection increases opportunities of microbial dysbiosis, which may perform a part in increased susceptibility to fatty liver diseases. However, it is demonstrated that probiotic prevented the diminished bacterial diversity and a severe gut microbiota alteration, which is consistent with Zhou et al.[37] who reported that probiotic treatment could prevent NAFLD progression via balance the gut microbiota. In the present study, the abundance of Proteobacteria was markedly enhanced by CORT exposure, and similar result has been reported by Hamid et al.[33], who noted that non-alcoholic steatohepatitis (NASH) subjects exhibited an increased abundance of Proteobacteria in laying hens. A study have shown that more abundance of Proteobacteria was found in fecal of children who were high-fat, and lowfiber consumers[38]. Notably, Intestinimonas, a short-chain fatty acids (SCFA) -producing intestinal bacterium[22], was enriched in the CORT\&PB group. It has been reported that SCFA is generally considered to 
have many important effects on maintaining host health such as providing nutrients and energies for the host[39], and reduced production of SCFA is a crucial pathogenic cause in fatty liver disease[40]. Our findings were consistent with these studies, indicating that probiotic supplementation could enrich the relative abundance of SCFA producers and change the proportion of the gut microbiota[41, 42].

\section{Conclusion}

We demonstrate for the first time that supplementation of probiotic to chicks had a beneficial effect on preventing fatty liver disease through regulating lipogenic genes expression and improving gut microbial balance. Thus, our results indicate that probiotic could represent a potential nutritional agent for preventing fatty liver disease in chickens.

\section{Tables}


Table 1

Ingredients and nutrient composition of diets.

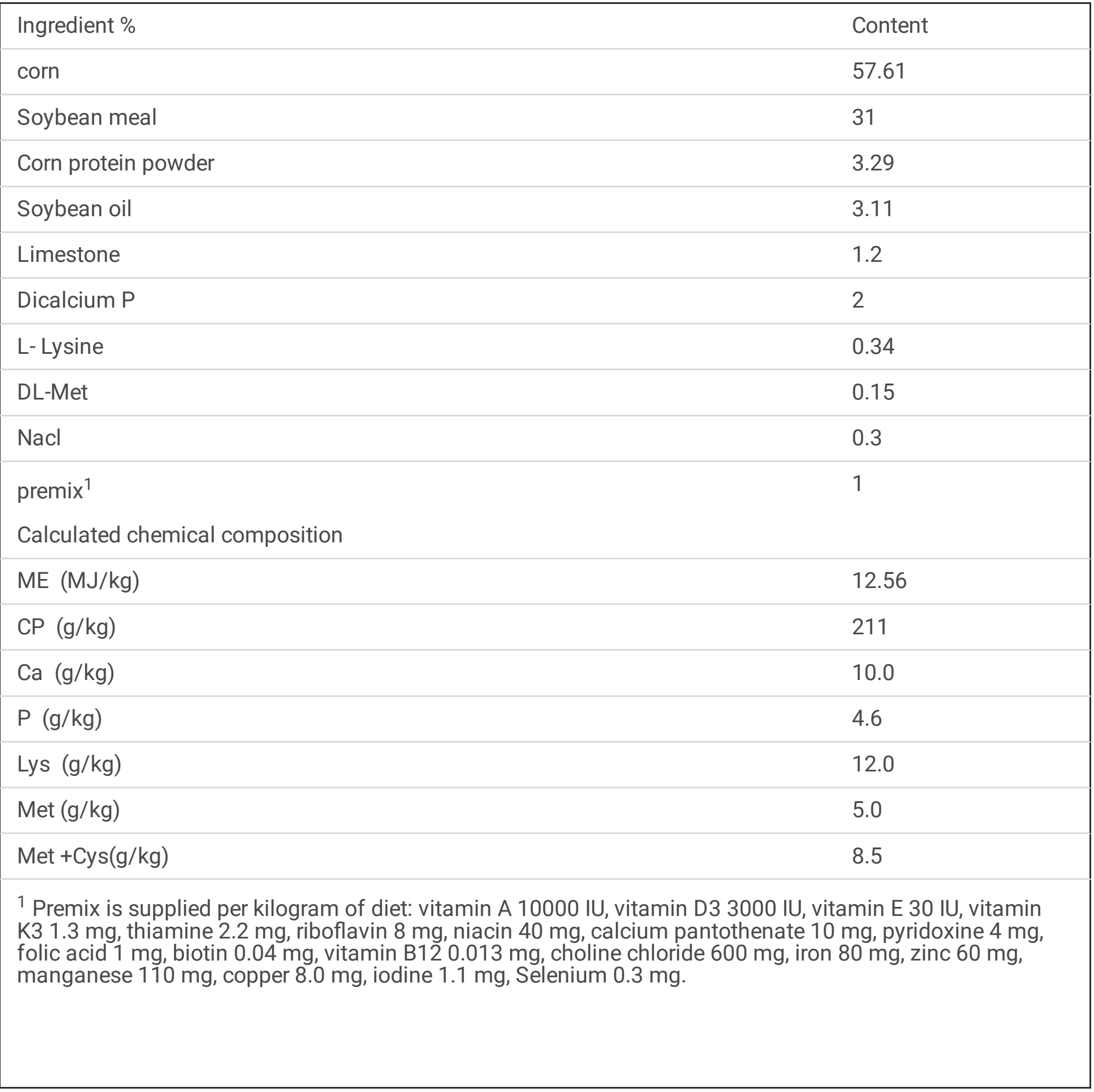


Table 2

PCR primers sequence.

\begin{tabular}{|c|c|c|c|}
\hline genes & Genbank accession & Primer sequences ( 5 ' to 3 ') & Fragment size \\
\hline \multirow[t]{2}{*}{ ACC } & \multirow[t]{2}{*}{ NM_205505.1 } & F: TGTGGGCTTTAGGAGATAA & 136 \\
\hline & & R:GGAACATTCAGGATACGC & \\
\hline \multirow[t]{2}{*}{ APOA1 } & NM_205525.4 & F: ATGCCATCGCCCAGTTCG & 165 \\
\hline & & R: GCAGAGCCTCGGTGTCCTT & \\
\hline \multirow[t]{2}{*}{ APOB } & NM_001044633.1 & F: CAGAGGTAGAGGCAGGAC & 82 \\
\hline & & R: TCATCGGAGAAGTTAGGA & \\
\hline \multirow[t]{2}{*}{ CD36 } & NM_001030731.1 & F:GCATCATTTCCTCCATTT & 110 \\
\hline & & R:ATTCCCTTCACGGTCTTA & \\
\hline \multirow[t]{2}{*}{ DGAT2 } & XM_419374.6 & F: GCTCTTCTCCTCGAACACG & 184 \\
\hline & & R: CAACCCGAACCTGCCTTT & \\
\hline \multirow[t]{2}{*}{ FASN } & NM_205155.3 & F:GGGAATGTCACACCTTGCTC & 164 \\
\hline & & R:GGAAATGGGTATTGTCGCTC & \\
\hline \multirow[t]{2}{*}{ LPL } & NM_205282.1 & F:CGGTGACAGGAATGTATGA & 140 \\
\hline & & R:CTTCGTGTAAGCAGCAGA & \\
\hline \multirow[t]{2}{*}{ PPARa } & AF163809.1 & F: TTGTCGCTGCCATCATTT & 147 \\
\hline & & R: GAAGTTTCGGGAAGAGGA & \\
\hline \multirow[t]{2}{*}{ PPARY } & NM_001001460.1 & F: CAGTGCAGGAGATTACAG & 87 \\
\hline & & R: CATATTTCAGGAGGGTTA & \\
\hline \multirow[t]{2}{*}{ SCD } & NM_204890.1 & F: GTTTCCACAACTACCACCAT & 173 \\
\hline & & R: ATCTCCAGTCCGCATTTT & \\
\hline \multirow[t]{2}{*}{ SREBP1 } & NM_204126.2 & F: GGCAGAGGAAGACAAAGGC & 123 \\
\hline & & R: AGCAGCAGTGACTCCGAGC & \\
\hline \multirow[t]{2}{*}{$\beta$-actin } & L08165.1 & F: CCCTGTATGCCTCTGGTC & 194 \\
\hline & & R: CTCGGCTGTGGTGGTGAA & \\
\hline
\end{tabular}


Table 3

Effect of probiotics and CORT on organs weight and indexes of broilers

\begin{tabular}{|c|c|c|c|c|c|c|c|}
\hline \multirow[t]{2}{*}{ Parameters } & \multirow[t]{2}{*}{ CON } & \multirow[t]{2}{*}{ CORT } & \multirow[t]{2}{*}{ PB } & \multirow[t]{2}{*}{ CORT\&PB } & \multicolumn{3}{|c|}{$P$-value } \\
\hline & & & & & CORT & PB & Interaction \\
\hline $\begin{array}{l}\text { Liver } \\
\text { weight(g) }\end{array}$ & $26.37 \pm 0.65^{b}$ & $32.71 \pm 1.83^{a}$ & $23.71 \pm 1.11^{b}$ & $32.16 \pm 1.78^{a}$ & 0.00 & 0.27 & 0.47 \\
\hline $\begin{array}{l}\text { Liver } \\
\text { index(\%) }\end{array}$ & $2.72 \pm 0.05^{b}$ & $3.54 \pm 0.17^{a}$ & $2.37 \pm 0.07^{b}$ & $3.28 \pm 0.16^{a}$ & 0.00 & 0.02 & 0.74 \\
\hline $\begin{array}{l}\text { Thymus } \\
\text { weight }\end{array}$ & $3.58 \pm 0.24^{b}$ & $0.91 \pm 0.07^{a}$ & $3.92 \pm 0.52^{b}$ & $1.30 \pm 0.12^{\mathrm{a}}$ & 0.00 & 0.25 & 0.93 \\
\hline $\begin{array}{l}\text { Thymus } \\
\text { index(\%) }\end{array}$ & $0.36 \pm 0.03^{b}$ & $0.11 \pm 0.01^{a}$ & $0.39 \pm 0.04^{b}$ & $0.13 \pm 0.01^{a}$ & 0.00 & 0.32 & 0.95 \\
\hline $\begin{array}{l}\text { Spleen } \\
\text { weight(g) }\end{array}$ & $1.84 \pm 0.09^{a c}$ & $1.28 \pm 0.09^{b}$ & $2.07 \pm 0.20^{a}$ & $1.55 \pm 0.17^{b c}$ & 0.00 & 0.11 & 0.88 \\
\hline $\begin{array}{l}\text { Spleen } \\
\text { index(\%) }\end{array}$ & $0.18 \pm 0.01^{\mathrm{ac}}$ & $0.14 \pm 0.01^{b}$ & $0.21 \pm 0.02^{a}$ & $0.16 \pm 0.02^{b c}$ & 0.00 & 0.14 & 0.94 \\
\hline $\begin{array}{l}\text { Fabricius } \\
\text { weight(g) }\end{array}$ & $3.08 \pm 0.19^{a}$ & $1.22 \pm 0.15^{\mathrm{b}}$ & $3.37 \pm 0.37^{a}$ & $1.30 \pm 0.11^{b}$ & 0.00 & 0.41 & 0.66 \\
\hline $\begin{array}{l}\text { Fabricius } \\
\text { index(\%) }\end{array}$ & $0.31 \pm 0.02^{a}$ & $0.12 \pm 0.01^{b}$ & $0.34 \pm 0.03^{a}$ & $0.13 \pm 0.01^{b}$ & 0.00 & 0.32 & 0.74 \\
\hline $\begin{array}{l}\mathrm{CON}, \text { control } \\
\text { treated grou } \\
\text { as means } \pm \\
0.05) \text {. }\end{array}$ & $\begin{array}{l}\text { oup; CORT, c } \\
\text { Organs index } \\
M(n=8) . D i\end{array}$ & $\begin{array}{l}\text { costerone-tre } \\
\text { are the ratio } \\
\text { ent small lett }\end{array}$ & $\begin{array}{l}\text { d group; PB } \\
\text { organs weigl } \\
\text { superscripts }\end{array}$ & $\begin{array}{l}\text { biotic-treate } \\
\text { lated to final } \\
\text { in a row indi }\end{array}$ & $\begin{array}{l}\text { roup; } \\
\text { ody we } \\
\text { te sign }\end{array}$ & $\begin{array}{l}\text { T\&P } \\
\text { t. Da } \\
\text { ant c }\end{array}$ & $\begin{array}{l}\text { CORT and PB } \\
\text { a are expressec } \\
\text { fferences }(P<\end{array}$ \\
\hline
\end{tabular}

\section{Abbreviations}

ACC

acetyl-CoA carboxylase; APOA1:Apolipoprotein A1; APOB:Apolipoprotein B; CD36:CD36 molecule; CORT, DGAT2:diacylglycerol O-Acyltransferase 2; FASN:fatty Acid Synthase; LPL:lipoprotein Lipase; PPARY:peroxisome proliferator activated receptor gamma; PPARa:peroxisome proliferator activated receptor alpha; SCD:stearoylCoA Desaturase; SREBP1:sterol regulatory element-binding protein-1.

\section{Declarations}

\section{Ethics approval and consent to participate}

The Animal Management and Ethics Committee of Nanjing Agricultural University (IACUC) approved all animal procedures. Sampling and slaughter producers were in accordance with "Guidelines on Ethical Treatment of Experimental Animals"(2006)No.398set by the Ministry of Science and Technology, China and 'Regulation 
regarding the Management and Treatment of Experimental Animals' (2008) No.45 formulated by the Jiangsu Provincial People's Government.

\section{Consent for publication}

Not applicable.

\section{Availability of data and material}

Not applicable.

\section{Competing interests}

The authors declare that they have no competing interests.

\section{Funding}

This work was funded by the National Key R\&D Program of China (2017YFE0114400) and Priority Academic Program Development of Jiangsu Higher Education Institutions(PAPD).

\section{Authors' contributions}

WM performed experiments and drafted the manuscript. YF ZY and HL: performed experiments and analyzed the data. YN and RZ: contributed to the experimental design and manuscript revision. YN: conceived the idea, designed the experiment, and finalized the manuscript. All authors read and approved the final manuscript.

\section{Acknowledgements}

The authors thank the National Key R\&D Program of China (2017YFE0114400), and the Priority Academic Program Development of Jiangsu Higher Education Institutions (PAPD).

\section{References}

1. Cohen JC, Horton JD, Hobbs HH. Human Fatty Liver Disease: Old Questions and New Insights. Science. 2011;332(6037):1519-23.

2. Angulo P. Nonalcoholic Fatty Liver Disease; 2008.

3. Choi SS, Diehl AM. Hepatic triglyceride synthesis and nonalcoholic fatty liver disease. Curr Opin Lipidol. 2008;19(3):295.

4. Paglialunga S, Dehn CA. Clinical assessment of hepatic de novo lipogenesis in non-alcoholic fatty liver disease. Lipids Health Dis. 2016;15(1):159.

5. John K, Marino JS, Sanchez ER, Hinds TD. The glucocorticoid receptor: cause of or cure for obesity? Am J Physiol-Endoc M. 2016;310(4):E249-57. 
6. Pasieka AM, Rafacho A. Impact of Glucocorticoid Excess on Glucose Tolerance: Clinical and Preclinical Evidence. Metabolites. 2016;6(3).

7. Buettner C. Mechanisms of Glucocorticoid-Induced Insulin Resistance Focus on Adipose Tissue Function and Lipid Metabolism. Endocrin Metab Clin. 2014;43(1):75-102.

8. Mei WQ, Hao YR, Xie HL, Ni YD, Zhao RQ. Hepatic Inflammatory Response to Exogenous LPS Challenge is Exacerbated in Broilers with Fatty Liver Disease. Animals. 2020;10(3):514.

9. Hu Y, Sun QW, Liu J, Jia YM, Cai DM, Idriss AA, et al. In ovo injection of betaine alleviates corticosteroneinduced fatty liver in chickens through epigenetic modifications. Sci Rep. 2017;7:40251.

10. Hu Y, Sun QW, Hu Y, Hou Z, Zong YB, Omer NA, et al. Corticosterone-induced Lipogenesis activation and Lipophagy inhibition in chicken liver are alleviated by maternal Betaine supplementation. J Nutr. 2018;148(3):316-25.

11. Usami M, Miyoshi M, Yamashita H. Gut microbiota and host metabolism in liver cirrhosis. World J Gastroenterol. 2015;21(41):123-34.

12. lacono A, Raso GM, Canani RB, Calignano A, Meli R. Probiotics as an emerging therapeutic strategy to treat NAFLD: focus on molecular and biochemical mechanisms. J Nutr Biochem. 2010;22(8):699-711.

13. Yang Q. Oral Administration of Compound Probiotics Ameliorates HFD-Induced Gut Microbe Dysbiosis and Chronic Metabolic Inflammation via the G Protein-Coupled Receptor 43 in Non-alcoholic Fatty Liver Disease Rats. Probiotics Antimicro. 2019;11(1):175-85.

14. Xin JG, Zeng D, Wang HS, Ni XQ, Yi D, Pan KC, et al. Preventing non-alcoholic fatty liver disease through Lactobacillus johnsonii BS15 by attenuating inflammation and mitochondrial injury and improving gut environment in obese mice. Appl Microbiol Biot. 2014;98(15):6817-29.

15. Cortez-Pinto H, Borralho P, Machado J, Lopes MT, Gato IV, Santos AM, et al. Microbiota Modulation With Synbiotic Decreases Liver Fibrosis in a High Fat Choline Deficient Diet Mice Model of Non-Alcoholic Steatohepatitis (NASH). Ge Portuguese Journal of Gastroenterology. 2016;23(3):132-41.

16. Wang K, Liao MF, Zhou N, Bao L, Ma K, Zheng ZY, et al. Parabacteroides distasonis Alleviates Obesity and Metabolic Dysfunctions via Production of Succinate and Secondary Bile Acids. Cell Reports. 2019;26(1):222-35.e225.

17. Ritze Y, Bárdos G, Claus A, Ehrmann V, Bergheim I, Schwiertz A, et al. Lactobacillus rhamnosus GG Protects against Non-Alcoholic Fatty Liver Disease in Mice. Plos One. 2014;9(1):e80169.

18. Yalçin S, Güçer Ş, Yalçin S, Onbaşilar İ, Kale G, Coşkun T. Effects of probiotic (Primalac 454) on nonalcoholic fatty liver disease in broilers. Revue Méd Vét. 2011;162(7):371-6.

19. Livak KJ, Schmittgen TD. Analysis of relative gene expression data using real-time quantitative PCR and the $2-\Delta \Delta$ CT method. methods. 2001;25(4):402-8.

20. Sun L, Dong H, Zhang Z, Liu J, Zhao R. Activation of epithelial proliferation induced by Eimeria acervulina infection in the duodenum may be associated with cholesterol metabolism. Oncotarget. 2014;7(19):27627-40.

21. Panchal SK, Poudyal H, lyer A, Nazer R, Brown L. High-carbohydrate, high-fat diet-induced metabolic syndrome and cardiovascular remodeling in rats. J Cardiovasc Pharm. 2011;57(5):611-24.

22. Li L, Guo WL, Zhang W, Xu JX, Qian M, Bai WD, et al. Grifola frondosa polysaccharides ameliorate lipid metabolic disorders and gut microbiota dysbiosis in high-fat diet fed rats. Food Funct. 2019;10(5):2560- 
72.

23. Lin H, Sui S, Jiao H, Buyse J, Decuypere E. Impaired development of broiler chickens by stress mimicked by corticosterone exposure. Comp Biochem Phys A. 2006;143(3):400-5.

24. Jin LZ, Ho YW, Abdullah N, Jalaludin S. Digestive and bacterial enzyme activities in broilers fed diets supplemented with Lactobacillus cultures. Poult Sci. 2000;79(6):886-91.

25. Kalavathy R, Abdullah N, Jalaludin S, Ho YW. Effects of Lactobacillus cultures on growth performance, abdominal fat deposition, serum lipids and weight of organs of broiler chickens. Br Poult Sci. 2003;44(1):139-44.

26. Wang Y, Wu YP, Wang BK, Xu H, Mei XQ, Xu XG, et al. Bacillus amyloliquefaciens SC06 Protects Mice Against High-Fat Diet-Induced Obesity and Liver Injury via Regulating Host Metabolism and Gut Microbiota. Front Microbiol. 2019;10:12.

27. Zhao NN, Yang S, Jia YM, Sun B, He B, Zhao RQ. Maternal betaine supplementation attenuates glucocorticoid-induced hepatic lipid accumulation through epigenetic modification in adult offspring rats. $J$ Nutr Biochem. 2018;54:105-12.

28. Leveille GA. In vitro hepatic lipogenesis in the hen and chick. Comp Biochem Phys. 1969;28(1):431-5.

29. Kemper JK, Choi S-E, Kim DH. Sirtuin 1 deacetylase: a key regulator of hepatic lipid metabolism. Vitamins hormones. 2013;91:385-404.

30. Donnelly KL, Smith Cl, Schwarzenberg SJ, Jessurun J, Boldt MD, Parks EJ. Sources of fatty acids stored in liver and secreted via lipoproteins in patients with nonalcoholic fatty liver disease. J Clin Invest. 2005;115(5):1343-51.

31. Wang HS, Ni XQ, Qing XD, Zeng D, Luo M, Liu L, et al. Live Probiotic Lactobacillus johnsonii BS15 Promotes Growth Performance and Lowers Fat Deposition by Improving Lipid Metabolism, Intestinal Development, and Gut Microflora in Broilers. Front Microbiol. 2017;8:14.

32. Lau E, Carvalho D, Freitas P. Gut Microbiota: Association with NAFLD and Metabolic Disturbances. Biomed Res Int. 2015;2015:1-9.

33. Hamid H, Zhang JY, Li WX, Liu C, Li ML, Zhao LH, et al. Interactions between the cecal microbiota and nonalcoholic steatohepatitis using laying hens as the model. Poult Sci. 2019.

34. Gordon JI. Microbial ecology: Human gut microbes associated with obesity. Nature. 2006;444(7122):10223.

35. Turnbaugh PJ, Ley RE, Mahowald MA, Magrini V, Mardis ER, Gordon JI. An obesity-associated gut microbiome with increased capacity for energy harvest. Nature. 2006;444(7122):1027-131.

36. Chen $\mathrm{CH}$, Huang MH, Yang JC, Nien CK, Yang CC, Yeh YH, et al. Prevalence and Risk Factors of Nonalcoholic Fatty Liver Disease in an Adult Population of Taiwan: Metabolic Significance of Nonalcoholic Fatty Liver Disease in Nonobese Adults. J Clin Gastroenterol. 2006;40(8):745-52.

37. Zhou YL, Zheng TY, Chen HT, Li YQ, Huang HL, Chen WJ, et al. Microbial Intervention as a Novel Target in Treatment of Non-Alcoholic Fatty Liver Disease Progression. Cell Physiol Biothem. 2018;51(5):2123-35.

38. De Filippo C, Cavalieri D, Di Paola M, Ramazzotti M, Poullet JB, Massart S, et al. Impact of diet in shaping gut microbiota revealed by a comparative study in children from Europe and rural Africa. P Natl Acad Sci USA. 2010;107(33):14691-6. 
39. den Besten G, van Eunen K, Groen AK, Venema K, Reijngoud D-J, Bakker BM. The role of short-chain fatty acids in the interplay between diet, gut microbiota, and host energy metabolism. $J$ Lipid Res.

2013;54(9):2325-40.

40. Schnabl B, Brenner DA. Interactions Between the Intestinal Microbiome and Liver Diseases. Gastroenterology. 2014;146(6):1513-24.

41. Jiao N, Baker SS, Nugent CA, Tsompana M, Cai LT, Wang Y, et al. Gut microbiome may contribute to insulin resistance and systemic inflammation in obese rodents: a meta-analysis. Physiol Genomics. 2018;50(4):244-54.

42. de LeBlanc AD, LeBlanc JG. Effect of probiotic administration on the intestinal microbiota, current knowledge and potential applications. World J Gastroentero. 2014;20(44):16518-28.

\section{Figures}

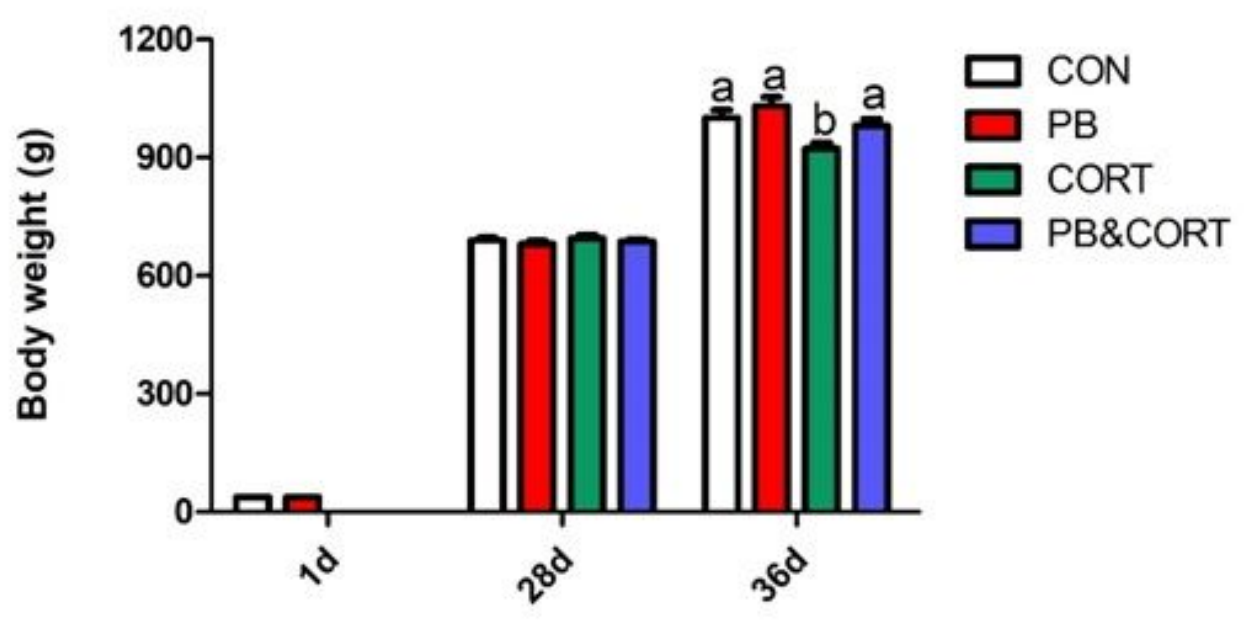

Figure 1

Effect of probiotic and CORT on body weight of broilers. CON, control group; PB, probiotic-treated group; CORT, corticosterone-treated group; PB\&CORT, PB and CORT-treated group. Data are expressed as means \pm SEM ( $\mathrm{n}=$ 8). Different small letter superscripts a,b indicate significant differences $(P<0.05)$. 


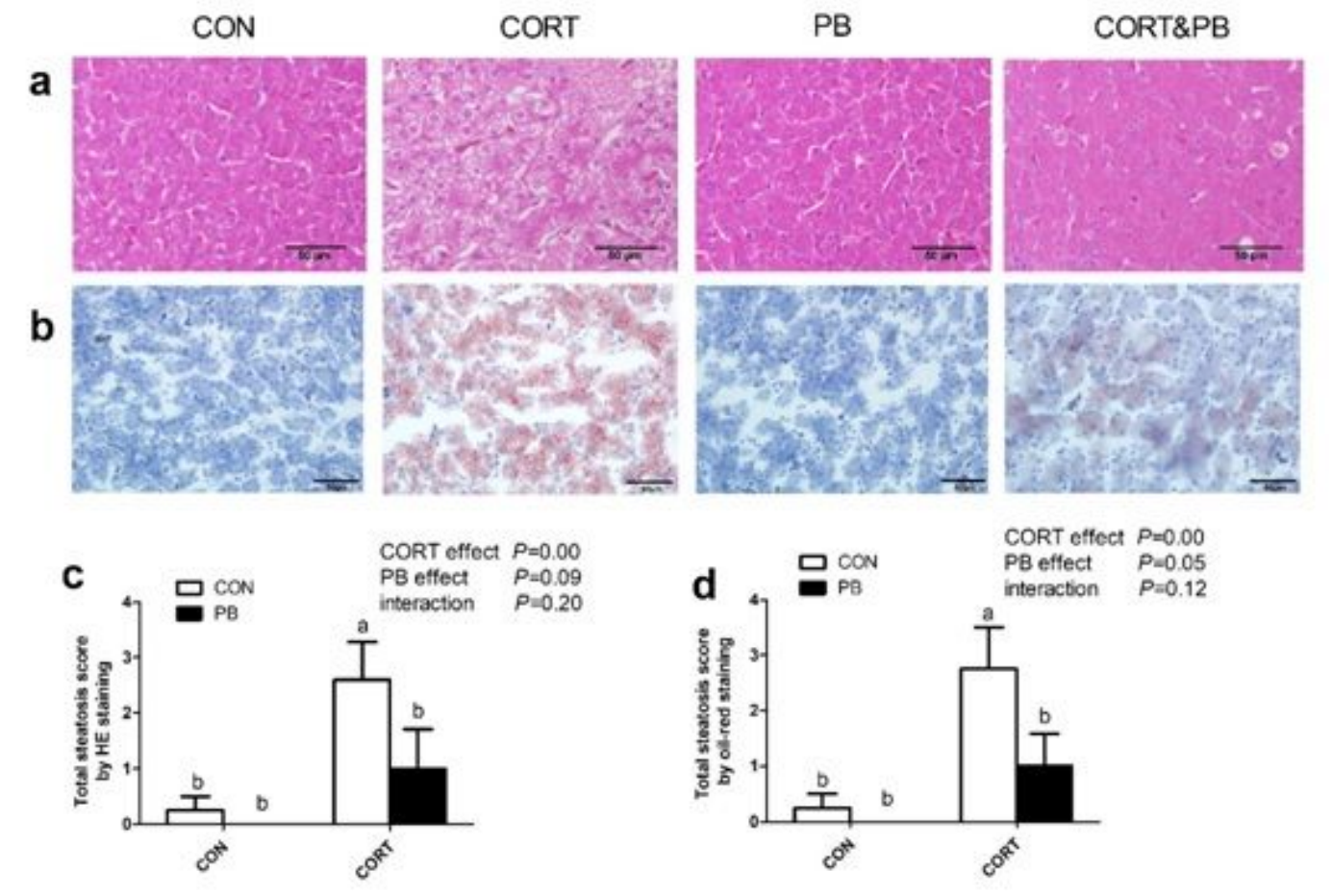

Figure 2

Effect of probiotic and CORT on hepatic hematoxylin-eosin $(\mathrm{HE})$ staining $(\times 200)$ and oil-red $(\times 400)$ staining of broilers. a, HE staining; b, Oil-red staining; c, Total steatosis score by HE staining; d, Total steatosis score by oilred staining. CON, control group; CORT, corticosterone-treated group; PB, probiotic-treated group; CORT\&PB, CORT and PB-treated group. Data are expressed as means \pm SEM $(n=5)$. Different small letter superscripts $a, b$ indicate significant differences $(P<0.05)$.
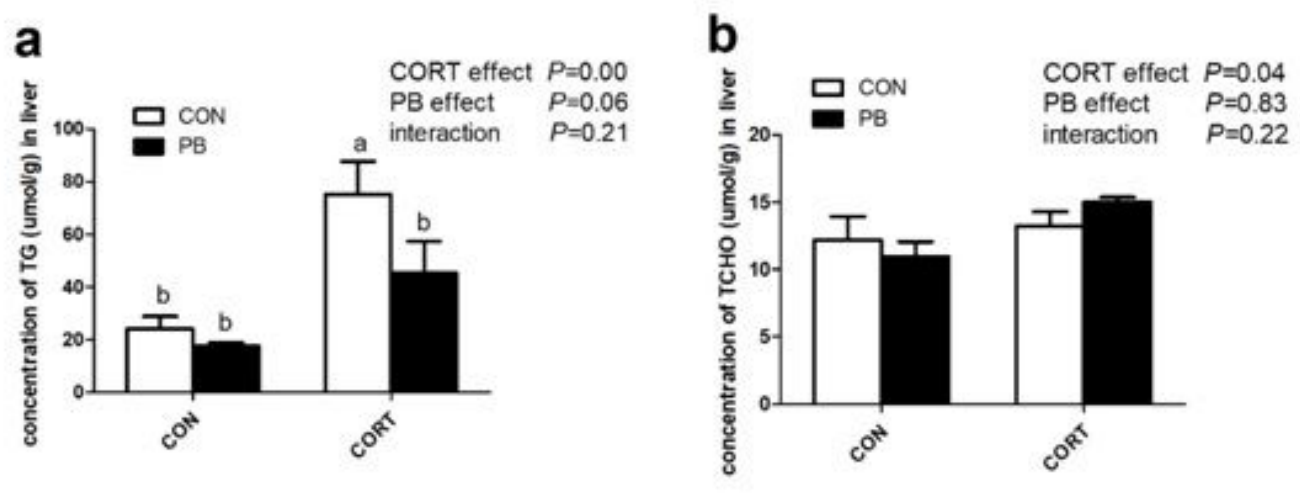

\section{Figure 3}

TG content in plasma and liver. a, TG content in plasma; b, TG content in plasma. C-CON, control group; C-CORT, corticosterone-treated group; P-CON, PB-treated group; P-CORT, PB- and CORT-treated group. Data are expressed as means $\pm \operatorname{SEM}(n=5)$. Different small letter superscripts $a, b$ in a row indicate significant differences $(P<$ 0.05). 



Figure 4

Effect of probiotic and CORT on liver expression of genes related to lipogenesis. a, Hepatic ACC mRNA expression; b, Hepatic SREBP1 mRNA expression; c, Hepatic FASN mRNA expression; d, Hepatic SCD mRNA expression; e, Hepatic PPARamRNA expression; f, Hepatic DGAT2 mRNA expression; g, Hepatic LPL mRNA expression; h, Hepatic PPARa mRNA expression; i, Hepatic CD36 mRNA expression; j, Hepatic APOA1 mRNA expression; $k$, Hepatic APOB mRNA expression; C-CON, control group; C-CORT, corticosterone-treated group; PCON, PB-treated group; P-CORT, PB- and CORT-treated group. Data are expressed as means \pm SEM $(n=8)$. Different small letter superscripts a-c indicate significant differences $(P<0.05)$. 

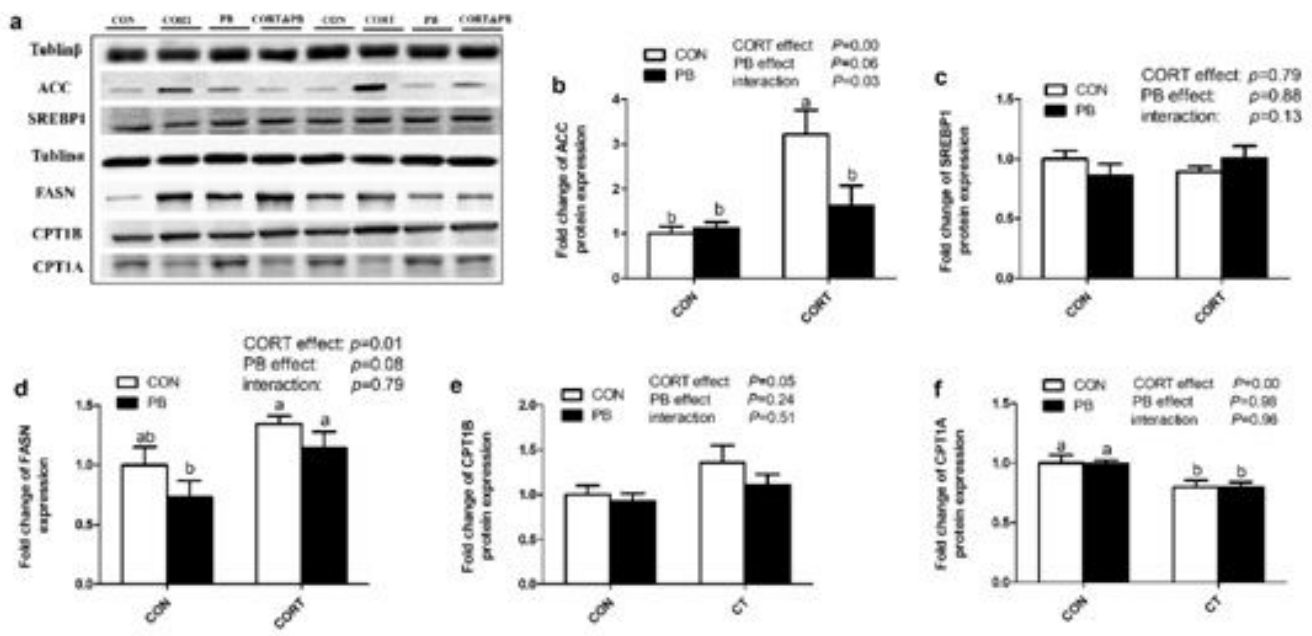

\section{Figure 5}

Effect of probiotic and CORT on liver expression of genes related to lipolysis. a, electrophoretogram; b, Hepatic ACC protein expression; c, Hepatic SREBP1 protein expression; d, Hepatic FASN mRNA expression; e, Hepatic CPT1B mRNA expression; f, Hepatic CPT1A mRNA expression;C-CON, control group; C-CORT, corticosteronetreated group; P-CON, PB-treated group; P-CORT, PB- and CORT-treated group. Data are expressed as means \pm SEM $(n=8)$. Different small letter superscripts a-c indicate significant differences $(P<0.05)$.

a

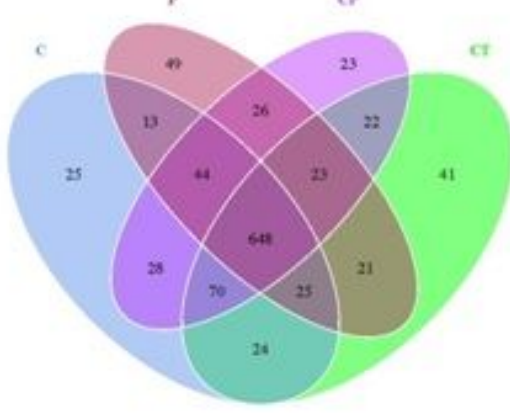

c

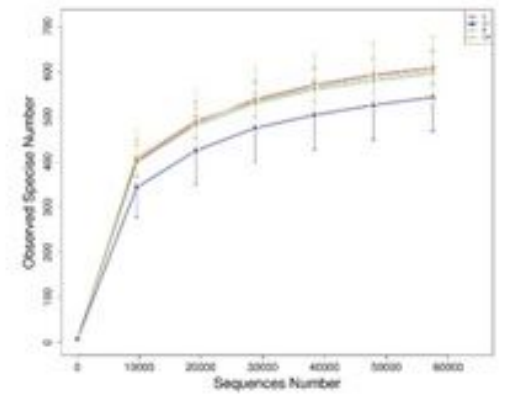

b



Figure 6

a, Venn Graph; b, Relative abundance of species at phylum level; c, Rarefaction Curve. C, control group; CT, corticosterone-treated group; P, probiotic-treated group; CP, CORT-and PB- treated group. $\mathrm{n}=4$. 

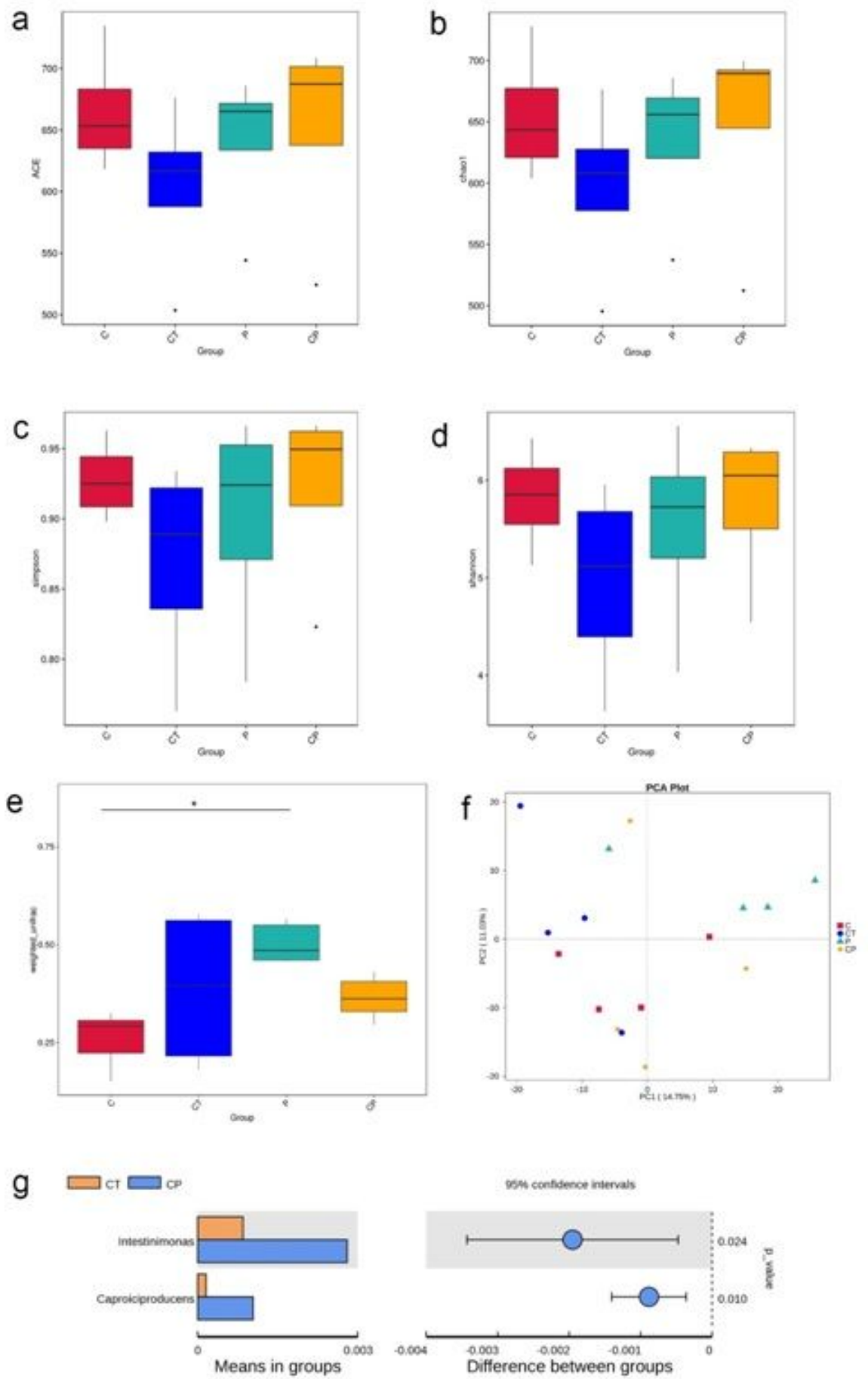

Figure 7

Alpha and Beta diversity analysis. The bacterial richness estimated by ACE index(a) andChao1 index (b);The bacterial diversity estimated by the Shannon index(c) and Simpson index(d).e, Beta diversity based on weighted unifrac; f,Principal Component Analysis(PCA); g, Differences species analysis by T-test. C, control group; CT, corticosterone-treated group; P, probiotic-treated group; CP, CORT-and PB- treated group. $\mathrm{n}=4$. 